\title{
RELAÇÃO ENTRE A TEMPERATURA DA SUPERFÍCIE DO MAR E A CAMADA DE MISTURA OCEÂNICA SOB A PASSAGEM DE CICLONES EXTRATROPICAIS NO ATLÂNTICO SUDOESTE
}

\author{
ANA CRISTINA PINTO DE ALMEIDA PALMEIRA ${ }^{1}$, RICARDO DE CAMARGO², \\ RONALDO MAIA DE JESUS PALMEIRA ${ }^{3}$
}

${ }^{1}$ Universidade Federal do Rio de Janeiro, Instituto de Geociências, Departamento de Meteorologia, Rio de Janeiro, RJ, Brasil

${ }^{2}$ Universidade de São Paulo, Instituto de Astronomia, Geofísica e Ciências Atmosféricas, São Paulo, SP, Brasil

${ }^{3}$ Universidade Federal do Rio de Janeiro, Instituto Alberto Luiz Coimbra de Pós-Graduação e Pesquisa de Engenharia, Rio de Janeiro, RJ, Brasil

anactn@gmail.com, ricamarg@model.iag.usp.br, palmeira@gmail.com

Recebido Junho de 2013 - Aceito Julho de 2014

\begin{abstract}
RESUMO
Neste artigo investigou-se a importância da variação da camada de mistura oceânica $(\mathrm{CMO})$ na temperatura da superfície do mar (TSM), sob a influencia de ciclones extratropicais atmosféricos. Um modelo simplificado de CMO oriundo do modelo HYCOM (Hybrid Coordinate Ocean Model), baseado no modelo de Kraus e Turner, foi inserido como uma sub-rotina do modelo BRAMS (Brazilian Regional Atmospheric Modelling System), para atualizar a TSM a cada passo de tempo no modelo atmosférico. A CMO-BRAMS caracterizou-se por estreitar durante as trajetórias das baixas pressões, embora não tenha indicado um significativo aprofundamento seguindo o anticiclone da retaguarda do sistema frontal. A TSM calculada pela CMO do BRAMS apresentou grande variação $\left(1^{\circ} \mathrm{C}-5^{\circ} \mathrm{C}\right)$ nos instantes iniciais. Entretanto, uma vez ajustada houve pouca variação da TSM no decorrer do tempo $\left(1,0^{\circ} \mathrm{C}-2,5^{\circ} \mathrm{C}\right)$, com o aprofundamento (estreitamento) da $\mathrm{CMO}$ associada à diminuição (aumento) à temperatura.

Palavras-chave: Camada de mistura oceânica; TSM; Ciclones do Atlântico Sul; Modelagem numérica; BRAMS; HYCOM.
\end{abstract}

ABSTRACT: RELATIONSHIP BETWEEN SEA SURFACE TEMPERATURE AND OCEANIC MIXED LAYER ON THE PASSAGE OF EXTRATROPICAL CYCLONES AT THE SOUTHWEST ATLANTIC

The importance of the oceanic mixed layer (OML) variation on the sea surface temperature (SST), under the influence of atmospheric extratropical cyclones was investigated in this paper. An OML simplified model derived from HYCOM (Hybrid Coordinate Ocean Model) model, based on Kraus and Turner model, was inserted as a subroutine on the BRAMS (Brazilian Regional Atmospheric Modelling System), to update the TSM every time step in the atmospheric model. The OML-BRAMS was characterized by narrowing during the trajectories of low pressure, but has not indicated a significant deepening following the anticyclone rear frontal system. The SST calculated by the BRAMS OML showed large variations $\left(1.0^{\circ} \mathrm{C}-5.0^{\circ} \mathrm{C}\right)$ at the initial times. However, as soon as SST has been adjusted, few variation over time occurs $\left(1.0^{\circ} \mathrm{C}-2.5^{\circ} \mathrm{C}\right)$, with the deepening (narrowing) of OML associated with decreasing (creasing) temperature.

Keywords: Oceanic mixed layer; SST; South Atlantic cyclones; Numerical modeling; BRAMS; HYCOM. 


\section{INTRODUÇÃO}

Uma das características mais marcantes do Atlântico Sudoeste é a presença da Confluência Brasil-Malvinas (CBM), que ocorre próxima à latitude $38^{\circ} \mathrm{S}$ e está situada ao longo das trajetórias das tempestades, a qual induz frentes, sistemas transientes e massas de ar frio dos oceanos Pacífico e Austral para o Atlântico Sul. Essa alta variabilidade dos fenômenos atmosféricos pode afetar às interações oceano-atmosfera (Campos et al., 1999). Trata-se de uma das regiões mais energéticas dos oceanos, originando uma forte frente termohalina. Ela é resultado da convergência da Corrente do Brasil (CB) quente (temperatura maior que $20^{\circ} \mathrm{C}$ ) e vinda de latitudes baixas com a Corrente das Malvinas (CM) fria (temperatura menor que $16^{\circ} \mathrm{C}$ ) e oriunda de altas latitudes, que torna-se menos salinas com a contribuição do estuário do Rio da Prata.

Wainer e Venegas (2002) identificaram a variação da posição da CBM devido à variação na intensidade dos ventos de oeste associados à porção sul do Anticiclone Subtropical do Atlântico Sul (ASAS) afetando o deslocamento da CM.

Em termos atmosféricos, a região é marcada pelo ASAS e pela passagem de sistemas, tanto transientes, quanto quase estacionários em qualquer época do ano e, como foco de interesse, os ciclones extratropicais.

Alguns autores investigaram os fluxos de calor e as anomalias da temperatura da superfície do mar (TSM) no Atlântico Sudoeste focando os ciclones extratropicais. Segundo Bosart e Lackmann (1995), os fluxos de calor latente e sensível oceânicos são cruciais nos primeiros estágios do ciclo de vida do ciclone.

Saraiva (1996) avaliou a passagem de um ciclone explosivo no Sudoeste do Atlântico através de modelagem numérica, e verificou que a liberação de calor sensível e latente da CBM além de ser fundamental para a rápida intensificação, modula a sua trajetória bem como a microfísica de nuvens baixas.

No intuito de avaliar padrões espaciais de TSM no Atlântico Sul, Wainer et al. (2003) detectaram diferenças nas intensidades dos fluxos de calor latente e sensível, resultantes de observações e resultados de modelagem climáticas. As variações na TSM foram detectadas desde a escala sub-sazonal até a interanual, influenciadas pelas interações na CBM.

Pezzi et al. (2005) verificaram que a camada limite atmosférica marinha (CLAM) é modulada pelo forte gradiente de TSM, e uma explicação para isso é baseada no fato de que a CLAM se auto-ajusta às modificações da TSM. Anomalias positivas de TSM induziriam as mudanças na estabilidade estática da CLAM. Nesse caso, o empuxo e a turbulência aumentariam sobre águas quentes e diminuiriam o cisalhamento vertical do vento na CLAM, gerando ventos mais fortes na superfície. $\mathrm{O}$ oposto seria esperado sobre águas frias.
Concluíram que a TSM de alta resolução melhorou as trajetórias dos sistemas.

No intuito de contribuir para o entendimento dos processos de interação oceano-atmosfera que ocorrem no Atlântico Sudoeste, este trabalho tem como objetivo comparar a evolução da TSM fornecida por um arquivo com valores constantes e outro arquivo de TSM, atualizado a cada passo de tempo, resultante da variação da espessura da camada de mistura oceânica (CMO) oriunda do modelo de circulação oceânica HYCOM, observando a importância da variação da CMO na TSM, sob a influência da passagem de ciclones extratropicais atmosféricos e as instabilidades associadas à eles.

Para tanto, foi utilizado um modelo simplificado de camada de mistura oceânica, inserindo-o a uma sub-rotina do modelo BRAMS, para avaliar a variação da TSM ativa (modificada) a cada passo de tempo e a variação dos fluxos de calor latente e sensível na interface ar-mar, investigando os mecanismos físicos envolvidos. Trata-se de uma investigação em modelagem numérica de mesoescala atmosférica e da relevância das variações temporais de curto prazo. O procedimento de inclusão da variação da TSM, na correção do balanço de energia em superfície, permite compreender melhor o comportamento dos processos de interface, além de avançar na questão do acoplamento termodinâmico entre modelos atmosféricos e oceânicos.

\section{MATERIAL E MÉTODOS}

Com o intuito de melhor compreender os processos da interface ar-mar, esta sessão concatena alguns aspectos físicos fundamentais de ambos os fluidos.

\subsection{Balanço de energia em uma camada oceânica}

O oceano não é uma superfície "ideal" (horizontalmente homogênea, plana, opaca a radiação, etc.), devido à presença de ondas, spray marinho, variações de turbidez, etc. Portanto, é mais apropriado considerar o balanço de energia em uma camada, a qual pode-se incluir algumas heterogeneidades de pequena escala. Essa camada deve possuir massa e capacidade térmica finitas para permitir que, sobre um dado intervalo de tempo, a energia possa ser armazenada ou liberada.

Se a superfície é relativamente lisa e homogênea de modo que a camada possa ser considerada limitada por dois planos horizontais (superior e inferior) pode-se escrever que (Figura 1):

$$
R_{n}=G-(H+L E)+D H
$$

onde: DH é a mudança na energia liberada / armazenada por unidade de tempo e de área da camada. Rn, (radiação líquida), $\mathrm{H}$ (fluxo de calor sensível) e LE (fluxo de calor latente) estão associados à interface superior enquanto $\mathrm{G}$ (fluxo de calor no oceano) à interface inferior (Figura 1). 


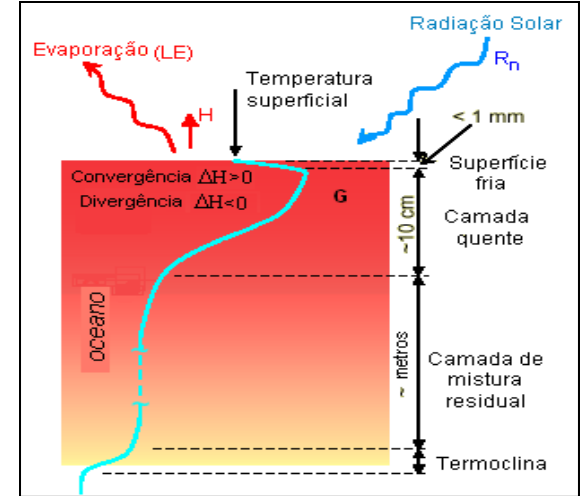

Figura 1: Balanço de calor na camada oceânica.

\subsection{Variação da temperatura da superfície do mar}

Para investigar os processos que levam a variação da TSM deve-se examinar a contribuição de calor na camada de mistura oceânica. O fluxo de calor líquido da superfície da atmosfera, para dentro da camada de mistura, é composto pela radiação de onda curta, onda longa, calor latente e sensível e a radiação que penetra na base da camada de mistura. Os processos oceânicos que contribuem para variações na temperatura da $\mathrm{CMO}$ são advecção zonal e meridional, entranhamento de água fria pela base da $\mathrm{CMO}$, e fluxo de calor turbulento vertical e meridional. O fluxo de calor turbulento zonal costuma ser desprezado após análise de escala.

Os termos de fluxo de calor na superfície para dentro da $\mathrm{CM}$ podem ser estimados usando fórmulas bulk. Essas relações são discutidas em muitas referências (Mc Phaden, 1982; Dobson e Smith, 1988; Weare, 1989).

Outro fator que interfere na TSM é a ressurgência, causando resfriamento da camada superficial oceânica e aumentando a concentração de nutrientes. As frentes oceânicas possuem um impacto sobre a estrutura da CLA marítima e esses gradientes de TSM, e mudam a distribuição horizontal dos fluxos ar-mar (Khalsa, 1989; Friehe et al., 1991). Frequentemente, observa-se ventos mais intensos no lado quente das frentes oceânicas em resposta aos fortes fluxos de calor turbulentos (Sweet et al., 1981). Entretanto, a aceleração não é constante e organizada ou homogênea como mostrado por Weller et al. (1998). Uma consequência dessas inomogeneidades é a existência de circulações de mesoescala (Doyle e Warner, 1990), difíceis de evidenciar por serem embebidas em circulações de grande escala atmosférica (Giordani et al., 1998).

\subsection{Camada de mistura oceânica}

A Camada de mistura oceânica (CMO) é uma região completamente turbulenta da camada superior do oceano e faz fronteira com a interface ar-mar. Ela tem o papel de armazenar calor na primavera e no verão e ocorre por mistura direta pelo vento e indireta pelas ondas. Existem duas principais categorias de critério para a determinação da $\mathrm{CMO}^{1}$ : uma define a profundidade da camada isotérmica a partir do perfil da temperatura e assume que essa é a CMO $(\mathrm{h}=\mathrm{TSM}-\Delta \mathrm{T}$, onde $\Delta \mathrm{T}<0,5^{\circ} \mathrm{C}$ ) e a outra define a CMO a partir do perfil de $\rho\left(\mathrm{h}=\Delta \mathrm{zt}\right.$, onde $\left.\Delta \mathrm{zt}=\rho-1000 \mathrm{kgm}^{-3}\right)$.

A camada isotérmica $\mathrm{dT}=0,5^{\circ} \mathrm{C}$ não coincide obrigatoriamente com a camada de mesma densidade $\mathrm{d} \rho=$ $\mathrm{d} \rho(\mathrm{dT}, \mathrm{dS})$. A diferença entre as duas chama-se camada de barreira $(\mathrm{CBARR}=\mathrm{zT}-\mathrm{z} \rho)$ e possui um efeito no balanço de calor da $\mathrm{CMO}^{2}$.

Do ponto de vista oceânico, a resposta oceânica às heterogeneidades dos eventos atmosféricos é muito importante para modificar a CMO: aprofundamento da camada é geralmente associado aos eventos de vento (Bane e Osgood, 1989). O avanço do ar frio pode induzir mudança na circulação da frente oceânica profunda como sugerido por Adamec e Elsberry (1985a, 1985b), Nof (1983) ou Huang (1990). Recentemente, Caniaux e Planton (1998) analisaram a resposta da frente oceânica a uma forte tempestade durante o SEMAPHORE $^{3}$ e detectaram que a evolução da CMO ocorreu durante a tempestade. Além disso, a resposta da $\mathrm{CMO}$ a essa tempestade foi a mesma em cada lado da frente em termos de fluxos de calor. Retro-alimentações (ou feedbacks) são possíveis através das mudanças resultantes na mistura oceânica e na correspondente transferência de energia, às quais, eventualmente mudam a TSM e agem na camada limite atmosférica (CLA) marinha.

A formação e evolução da CMO são determinadas por mecanismos mecânicos e térmicos. O mecânico (fenômeno mais importante) é a mistura turbulenta devido a forçante atmosférica local na superfície da água, como por exemplo, o aumento da velocidade do vento que intensifica a mistura e aprofunda a CMO. O térmico é devido ao aquecimento (resfriamento) da superfície, enfraquecendo (intensificando) a mistura e obtendo uma CMO mais rasa (profunda).

Existe também a variação latitudinal. Nas baixas latitudes, a absorção da radiação solar é alta ${ }^{4}$, amortecendo a ECT da CMO e resultando em mistura fraca e CMO rasa (10-20 $\mathrm{m})$ enquanto que nas altas latitudes, o aquecimento é menor, a mistura pelo vento é forte e a CMO aprofunda (20-100 m).

${ }^{1}$ Vale ressaltar que a camada de Ekman é dinâmica e é diferente da CMO que é termodinâmica.

${ }^{2} \mathrm{~A}$ camada de barreira positiva é típica das regiões dos polos e dos trópicos, enquanto que nos subtrópicos, ela é negativa (Figura 2). Os casos de camada de barreira inexistente ocorrem quando o calor introduzido na CMO pela radiação é balanceado pelo frio do topo da termoclina. $\mathrm{O}$ entranhamento de água fria permite que a temperatura da $\mathrm{CMO}$ não aumente infinitamente.

${ }^{3}$ Structure des Echanges Air-Atmosphere, Propriétés des Heétérogénéités Océaniques: Recherche Expérimentale (SEMAPHORE experiment - Eymard et al., 1996), foi um experimento no Atlântico Norte no outono de 1993.

${ }^{4} \mathrm{O}$ fluxo líquido de calor é geralmente para baixo 
Sazonalmente, a camada de mistura profunda no inverno, e torna-se mais rasa no verão. Em escala diurna, a CMO é mais espessa à noite devido a forças atuantes e conjugadas.

Sabe-se que existe uma correlação da variação temporal de TSM (dTSM/dt) e a profundidade da CMO, dependendo das condições de superfície, existindo uma correlação positiva e outra negativa (Chu, 1993). Quando a superfície do oceano está sob ventos fracos, a CMO converge para a escala de comprimento de Monin-Obukhov, pois a ECT gerada pelo vento é insuficiente para empurrar a água profunda estável (fria e estratificada). Como o ganho líquido de calor na superfície do oceano é muito menor do que a perda de calor na base da CMO, leva ao aquecimento dela. Quanto mais fina (espessa) a CMO, mais ela aquece (resfria), deixando $\mathrm{dTSM} / \mathrm{dt}$ e a profundidade da CMO negativamente relacionadas.

Entretanto, sob ventos fortes, a $\mathrm{CMO}$ aprofunda devido ao aumento da ECT gerada pelo vento ser suficiente para empurrar a água fria na base da CMO. A excessiva perda de calor na base (entranhamento de fluxo de calor) sobre o ganho líquido de calor na superfície leva ao resfriamento da CMO. Quanto mais fina (espessa) a CMO, mais fria (quente), deixando a dTSM/dt e a profundidade positivamente correlacionadas.

\subsection{Modelo atmosférico BRAMS}

Para a parte atmosférica, foi escolhido o modelo BRAMS (Brazilian Regional Atmospheric Modeling System), na versão usada 5.04 plus (Fazenda et al., 2006).

Os casos simulados partiram da mesma configuração em seu namelist (RAMSIN): usando apenas uma grade regular, possuindo 145 pontos na direção leste-oeste (eixo x) e 166 pontos na direção norte-sul (eixo y) centrada em $27,5^{\circ} \mathrm{S} 045^{\circ} \mathrm{W}$, sendo o espaçamento entre os pontos de grade de $30 \mathrm{Km}$ em ambas as direções; ou seja, um domínio de aproximadamente $40^{\circ} \times 45^{\circ}(4350 \mathrm{Km} \times 4980 \mathrm{Km})$. Verticalmente, a discretização foi de $50 \mathrm{~m}$ até os primeiros $1000 \mathrm{~m}$ e a partir desse nível, utilizou-se uma taxa de espaçamento de 1,2. A condição inicial e as condições de contorno laterais e de topo consideraram dados da grande escala oriundos das Reanálises do NCEP a cada 6 horas, tendo sido utilizados 5 pontos com decaimento exponencial a partir da borda para as correspondentes especificações. As integrações foram de 168 horas (7 dias) com saídas a cada hora. As parametrizações de cumulus (Grell) e de radiação (Chen) foram ativadas, bem como as de microfísica (nível 3). Os coeficientes de difusão horizontal e vertical para esta grade foram baseados, respectivamente, na formulação de Smagorinsky e o esquema de Mellor e Yamada.

\subsection{Modelo de camada de mistura oceânica de Kraus e Turner}

Das inúmeras modelagens de CMO disponíveis na literatura, foi escolhido o modelo clássico de Kraus e Turner (1967), de modo a aproveitar uma rotina pública (mxkrtm.f) do modelo de circulação oceânica HYCOM (Hybrid Coordinate Ocean Model $)^{5}$, quando configurado no modo MICOM (Miami Isopycnal Coordinate Ocean Model), e já direcionar o presente trabalho ao ramo de pesquisa que utiliza simulações síncronas para investigar o acoplamento oceano-atmosfera. Outro fator importante para a escolha, além da relativa simplicidade desta CMO para inserir como subrotina do BRAMS, foi possibilidade de comparar as $\mathrm{CMO}$ oriundas das reanálises do próprio HYCOM com a CMO simulada pelo BRAMS: ambas usando Kraus-Turner.

O modelo Kraus e Turner (1967) (doravante K-T) considera explicitamente os processos de trocas oceanoatmosfera no contexto da teoria isopicnal.

\section{a. Formalismo e técnicas numéricas}

Em tal modelo de evolução de $\mathrm{CMO}$, o problema de fechamento das equações turbulentas é muito simplificado, sendo determinados os fluxos turbulentos nas fronteiras da CMO. Por isso, uma velocidade de entranhamento we é introduzida na base da camada tal que:

$$
w_{e}=\frac{d h}{d t} \text { se } \frac{d h}{d t}>0, w_{\mathrm{e}}=0 \text { se } \frac{d h}{d t} \leq 0
$$

Os fluxos turbulentos verticais na base da CMO por uma equação de forma geral são dados por:

$$
-\left(\overline{a^{\prime} w^{\prime}}\right)_{-h}=w_{e} \Delta a
$$

onde, $\Delta a$ descreve a descontinuidade da variável na base da camada. Nesse modelo, o efeito do vento é representado pelo stress superficial.

\section{b. Energia interna e Energia Cinética Turbulenta (ECT)}

A evolução da equação da energia interna de uma camada homogênea de temperatura $\theta$ s é dada por:

$$
h \frac{\partial \theta s}{\partial t}=\left(\overline{\theta^{\prime} w^{\prime}}\right)_{-h}-\left(\overline{\theta^{\prime} w^{\prime}}\right)_{0}+\frac{1}{\rho C_{p}}\left(R_{0}-R_{h}\right)-\left[K_{H}\left(\frac{\partial \theta}{\partial z}\right)\right]
$$

onde $-\left(\overline{\theta^{\prime} w^{\prime}}\right)_{0}=\frac{P}{\rho C p},-\left(\overline{\theta^{\prime} w^{\prime}}\right)_{-h}=w_{e} \Delta \theta$, $\mathrm{R}_{\mathrm{Z}}$ é a radiação solar na profundidade $\mathrm{z}$ e $\mathrm{P}$ é a perda de calor da superfície (radiação infravermelha, fluxos de calor sensível

${ }^{5}$ http://oceanmodeling.rsmas.miami.edu/hycom/overview.html. 
e latente). $\Delta \theta$ representa a descontinuidade da temperatura na base da CMO. O fluxo $\left[K_{H}(\partial \theta / \partial z)\right]_{0}$ é considerado na superfície e se este termo é nulo, está situado no limite superior da camada homogênea.
O conhecimento da velocidade de entranhamento necessita de uma equação complementar. $\mathrm{E}_{\mathrm{m}}$ um modelo de camada de mistura, esse cálculo é extraído usando a integração da equação da energia cinética turbulenta:

$$
\frac{1}{2} \int \frac{\partial E}{\partial t} d z=\left[\frac{E}{2}+\frac{P I^{\prime}}{R o 0} w^{\prime}\right] \text { pase }-\left[\frac{E}{2}+\frac{P I^{\prime}}{R o 0} w^{\prime}\right] s f c-\int u^{\prime} w^{\prime}(\partial u / \partial z) d z+\int b^{\prime} w^{\prime} d z-h E m
$$

(1)

(3)
(5) com: $\mathrm{u}(\mathrm{u}, \mathrm{v})$ : velocidade horizontal; $\mathrm{b}=\mathrm{g}(\mathrm{w} 0-\mathrm{w}) / \mathrm{w} 0$ : empuxo; PI: pressão e Em é a média da ECT dissipada em calor na CM.

$\mathrm{Na}$ forma completa, a Equação 5 expressa então o balanço entre: (1) a tendência de ECT contida na CMO, (2) o fluxo de ECT na base da CMO, (3) o fluxo de ECT na superfície, (4) o termo de produção de ECT devido ao cisalhamento da corrente oceânica, (5) o termo de consumo correspondendo ao empuxo da camada de mistura, podendo ser de destruição ou de produção de ECT e (6) o termo de dissipação de calor, onde h é calculado pelo modelo e equivale a distância vertical onde o gradiente vertical de temperatura torna-se diferente de zero.

\subsection{A inserção do módulo de CMO para diferenciar a TSM no BRAMS}

É importante ressaltar que esta metodologia não configura acoplamento de dois modelos, e sim a inserção de um módulo de CMO para variar a TSM de forma mais interativa do que simplesmente assimilar as informações disponíveis em base climatológica, semanal ou diária. Partindo dessa informação e sabendo das limitações relacionadas à ausência de dados oceanográficos na área de estudo, precisou-se de algum parâmetro para verificar o resultado da variação da altura da $\mathrm{CMO}$, que considerasse além dos fatores termodinâmicos, a contribuição da circulação. A saída para isso foi encontrada através da utilização do modelo oceânico HYCOM, que usa como modelo de CMO a parametrização de K-T, a mesma metodologia inserida como rotina no presente estudo.

Nas simulações do BRAMS com a CMO ativa, os dados de Climatologia do LEVITUS foram usados como perfil (estimativa inicial) para encontrar a altura da base da CMO e da termoclina, necessárias para modelo K-T. Após esta verificação, o valor da TSM climatológica do BRAMS foi comparado com os dados do LEVITUS e então foi feita uma diferença em todo o perfil para que a TSM no caso da CMO ativa, esteja ajustada à diferença do valor da climatológica e propague sua diferença de temperatura até a profundidade final da CMO (estimada pelo LEVITUS). Daí por diante (a partir da termoclina), o módulo assume o perfil climatológico LEVITUS, sem alterações. No próximo instante, a CMO ativada já apresentará um novo perfil, calculado pelo modelo K-T (Figura 2).

\subsection{Caracterização das situações de interesse consideradas nos experimentos numéricos}

A escolha da simulação foi fundamentada na disponibilidade de informações úteis e na relevância do fenômeno enfocado. Trata-se da região da CBM, onde o forte gradiente de TSM costuma intensificar os fluxos de calor em superfície e favorecer o abaixamento da pressão atmosférica.

No período de 01NOV a 08NOV2004, uma frente fria, associada a um ciclone extratropical $(992 \mathrm{hPa})$, atuou no sul da área (Figura 3), tendo como suporte dinâmico em níveis médios $(500 \mathrm{hPa})$ uma forte advecção de vorticidade negativa. Em superfície, verificou-se uma região marcada de calor latente (positiva) à Nordeste do sistema frontal, além de um núcleo de movimento ascendente - marcado pela variável Omega (velocidade vertical em $\mathrm{Pa} / \mathrm{s}$ ) um pouco mais a leste deste. Todo este padrão moveu-se rapidamente para leste, deixando um cavado em superfície e instabilizando a região. Vale ressaltar que a partir do dia 03NOV2004, o sistema avançou para leste associado aos núcleos de vorticidade relativa em 500 hPa, típico do avanço de um ciclone. Em níveis altos, notou-se ainda um um vórtice ciclônico (estrutura barotrópica) e a corrente de jatos favorecendo ainda mais o posicionamento da baixa pressão em superfície.

Durante a gênese e parte da trajetória inicial deste ciclone foram medidos os perfis de temperatura (e com isso a CMO) através de sondas XBT (batitermógrafos descartáveis) coletados

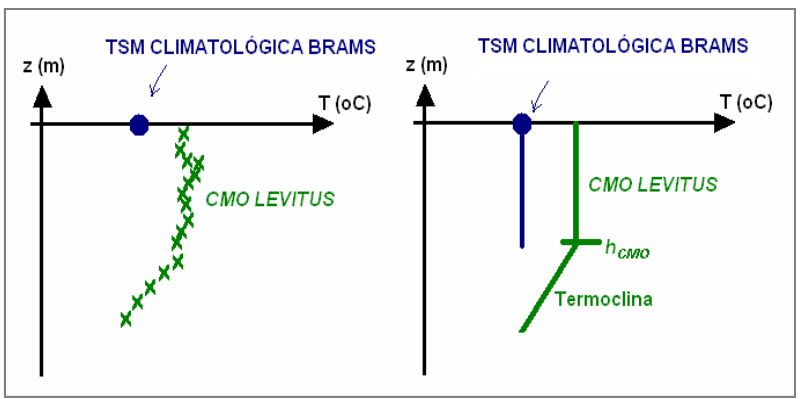

Figura 2: Metodologia da inserção do módulo de CMO no BRAMS. 


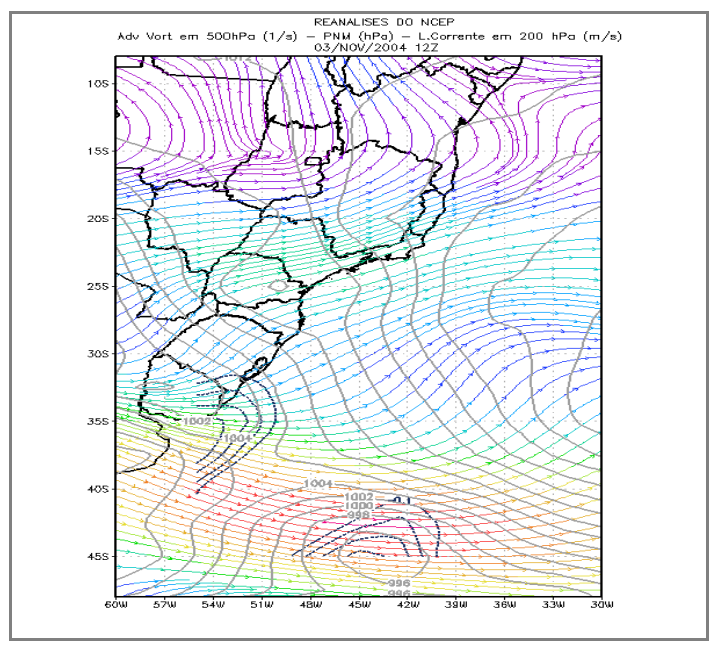

(a)

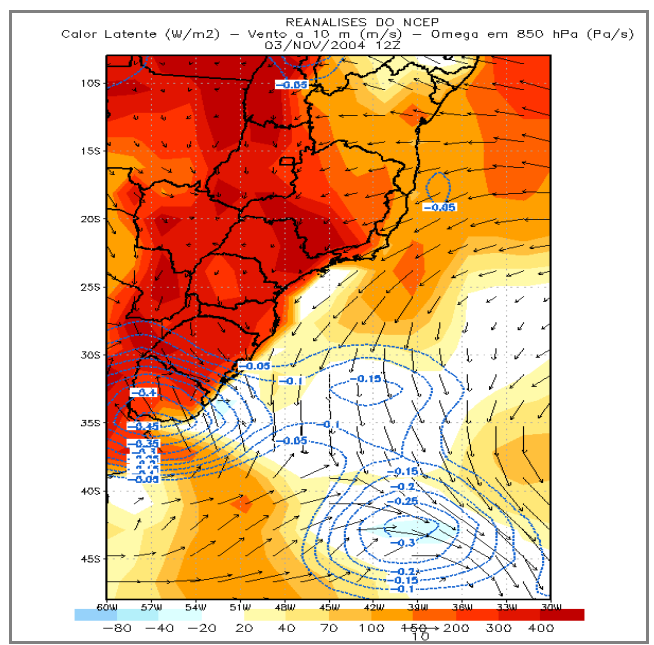

(b)

Figura 3: (a) Pressão ao nível médio do mar (linhas cinzas), advecção de vorticidade relativa em 500 hPa (pontilhados em azul escuro) e linhas de corrente em $200 \mathrm{hPa}$ (isolinhas coloridas) e (b) Fluxo de calor latente (sombreado), vento a 10 metros (vetores) e omega em $850 \mathrm{hPa}$ (isolinhas em azul escuro), a partir de dados das Reanálises do NCEP as 1200 Z em 03NOV2004.

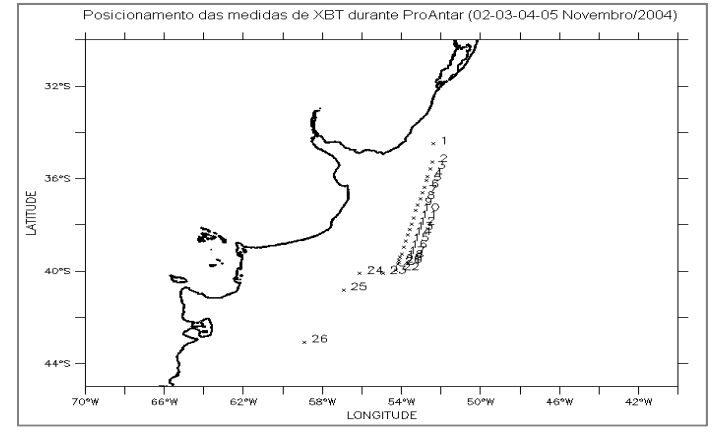

(a)

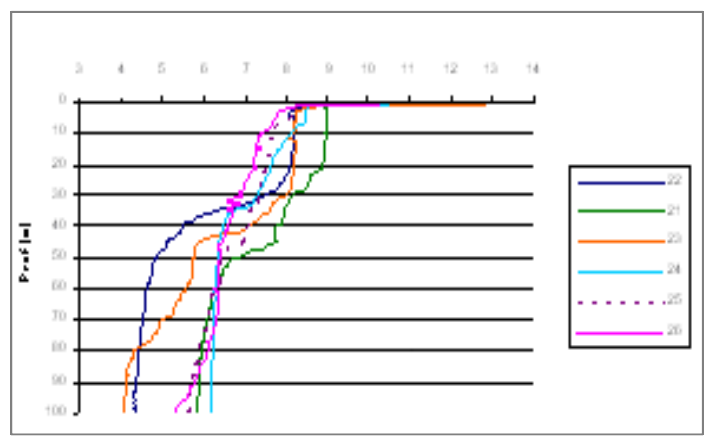

(b)

Figura 4: (a) Localização das sondagens com XBT no Sudoeste do Atlântico entre 02-05 de novembro de 2004. Os valores 1-26 indicam a ordenação cronológica de cada medida oceanográfica. (b) Perfis de temperatura $\left({ }^{\circ} \mathrm{C}\right)$ na região oeste do Atlântico Sul.

durante o cruzeiro do programa CNPq/ProAntar (Programa Antártico Brasileiro) em pontos durante a sua travessia desde o Rio de Janeiro até a Ilha Rei George (Antártica). Vale ressaltar ainda, que os dados foram utilizados apenas durante o período de 02-05 de novembro de 2004 (Figura 4), pois a partir desta data, o navio rumou para sul e suas medidas tornam-se irrelevantes para o ciclone em questão.

Mesmo considerando o curtíssimo período de amostragem e a região de complexa variabilidade oceânica da CBM, as medições de espessura da CMO, através dos perfis XBT observados no INTERCONF, permitiram alcançar estimativas muito úteis na inicialização de experimentos, apesar de suas abruptas descontinuidades espaciais. Foram encontrados, tipicamente, valores entre 20 e $100 \mathrm{~m}$, os quais possuem grande correspondência com outras fontes de informação sobre a CMO, como descrito na sequência.
Uma outra fonte de informação para os dados da altura da CMO é oriunda das simulações operacionais do Modelo HYCOM. Este modelo disponibiliza dados oceânicos (corrente, temperatura, salinidade, etc.) diariamente produzindo reanálises em formato NetCDF. Possui grade mercator entre $78^{\circ} \mathrm{S}$ e $47^{\circ} \mathrm{N}$ (resolução equatorial de $1 / 12^{\circ}$ ) e forçante atmosférica do Navy Operational Global Atmospheric Prediction System (NOGAPS), incluindo stress do vento, velocidade do vento, fluxo de calor e precipitação ${ }^{6}$.

Através da Figura 5 nota-se uma região (marcada no retângulo) mais rasa e quente, aprofundando e resfriando no decorrer da simulação. O retângulo vermelho indica a posição das medidas de XBT coletadas pelo navio; além de uma boa correspondência entre as simulações do HYCOM e as medidas

${ }^{6}$ http://hycom.coaps.fsu.edu/data/glb_nrt.html. 


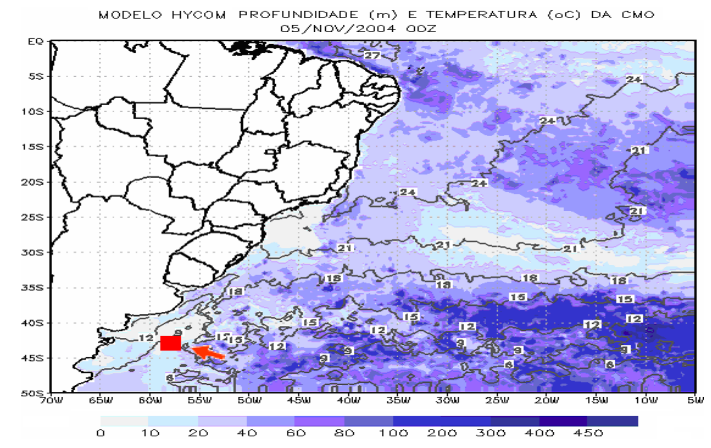

Figura 5: Profundidade e Temperatura da CMO segundo as simulações disponibilizadas do Modelo HYCOM.

in situ nos valores de TSM no dia 05NOV2004 com um padrão ainda mais frio $\left(7^{\circ} \mathrm{C}\right)$ e profundo $(>30 \mathrm{~m})$.

\section{RESULTADOS E DISCUSSÃO}

Em termos médios, verificou-se através do modelo BRAMS que as diferenças na TSM mantiveram-se aproximadamente constantes no decorrer da simulação, com núcleos negativos e positivos não ultrapassando $3^{\circ} \mathrm{C}$. É válido observar que as maiores discrepâncias localizam-se exatamente na região do Atlântico Sudoeste, onde as baixas pressões tendem a deslocar-se (Figura 6a-6c). Vale ressaltar ainda que a simulação com a CMO ativada no BRAMS apresentou pressões ligeiramente mais baixas em superfície (diferença de $1 \mathrm{hPa}$ entre os experimentos), uma precipitação mais representativa e uma nítida variação dos fluxos superficiais de calor latente (mais evidentes). O calor latente indicou um núcleo negativo (caso controle maior que no caso CMO-BRAMS) localizado entre $36^{\circ} \mathrm{S}-39^{\circ} \mathrm{S}$ e $046^{\circ} \mathrm{W}-050^{\circ} \mathrm{W}$; ou seja, próximo à alta pressão na retaguarda do sistema frontal. O campo de calor sensível apresentou maiores discrepâncias que o de calor latente, confirmado pelas diferenças de TSM e nem tanto pela advecção da temperatura do ar. A diferença entre o calor sensível das simulações também indicou maiores módulos que os de calor latente sobre o continente.

Para visualizar de forma mais clara a trajetória percorrida pelo primeiro ciclone que influenciou o domínio escolhido, foi plotada uma trajetória $\mathrm{AB}$ desde o seu inicio até sua posição final e observou-se que seu deslocamento foi aproximadamente zonal (Figura 7). Notou-se que a trajetória esteve em uma região de fronteira entre a camada de barreira dos subtrópicos (negativa) e a dos pólos (positiva); ou seja, podendo haver períodos de camada de barreira aproximadamente nula.

Com a finalidade de acompanhar a mesma coluna d'água antes e depois da passagem do ciclone, foi feito um perfil da variação da TSM e da espessura da CMO dentro da trajetória AB (Figura 8). Desta forma, verificou-se que o ciclone interferiu de maneira a ressurgir a coluna oceânica influenciando até as camadas mais profundas. Inicialmente (seta vermelha direcionando o ponto A), a CMO apresentava-se rasa $(50 \mathrm{~m})$ e fria $\left(7,0^{\circ} \mathrm{C}-8,0^{\circ} \mathrm{C}\right)$, para em seguida sofrer estreitamento, sem variar significativamente a sua temperatura. Vale ressaltar que a latitude na qual o ciclone se encontrava, possui climatologicamente uma TSM fria e que qualquer elevação de temperatura requer uma grande entrada de energia (CMO mais quente e mais profunda). Isto justifica a região ligeiramente mais quente próxima ao ponto $\mathrm{B}$.

A mesma metodologia foi aplicada para o segundo ciclone que seguiu com trajetória para SE, variando bastante latitudinalmente (Figura 9). Desta vez, mantendo o seu deslocamento dentro da região subtropical, com camada de barreira negativa.

Inicialmente (05NOV2004-18Z), o ciclone deslocou-se sobre uma TSM superior a $17^{\circ} \mathrm{C}$ com profundidade espessa $(200 \mathrm{~m})$, estreitando gradativamente $(100 \mathrm{~m})$ até o final de sua

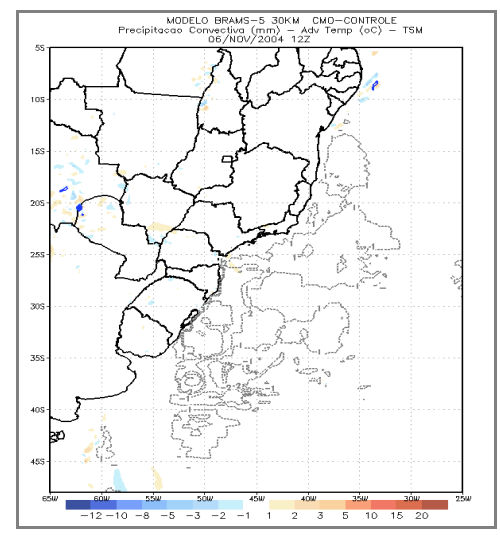

(a)

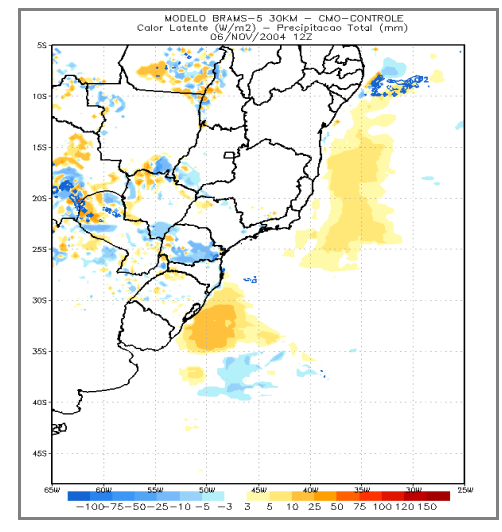

(b)

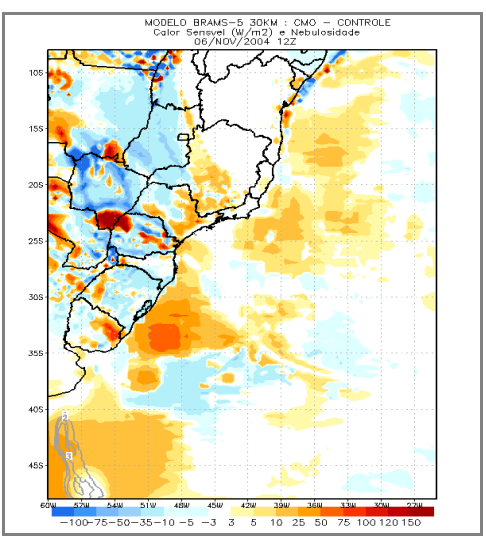

(c)

Figura 6: Diferenças entre os campos de (a) advecção de temperatura (sombreado), precipitação convectiva (isolinhas azuis) e TSM (em cinza); (b) calor latente e precipitação total e (c) calor sensível e nebulosidade simulados pelo modelo BRAMS. 


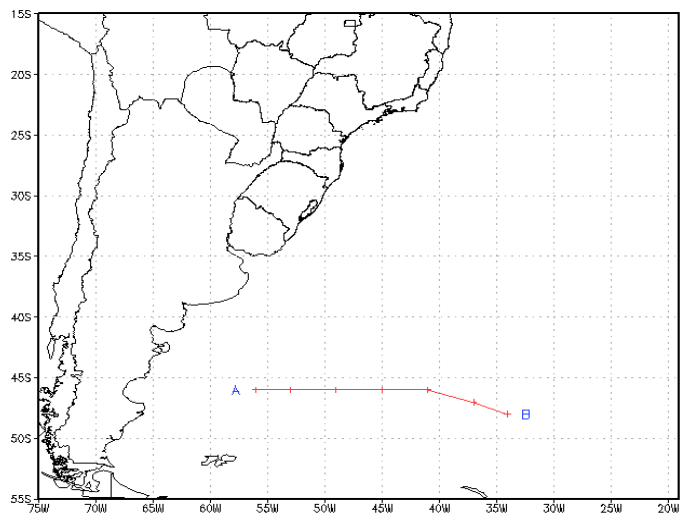

Figura 7: Trajetória do primeiro ciclone.

trajetória, além de um resfriamento de aproximadamente $3^{\circ} \mathrm{C}$ em 07NOV2004-12Z (Figura 10). Mais uma vez, nota-se que além da região da $\mathrm{CMO}$, a área da passagem do ciclone ficou marcada com uma variação em toda a coluna d'água, suspendendo as isolinhas de temperatura e adquirindo características das latitudes mais altas.

Com base nestes resultados, foram escolhidos dois pontos estratégicos para melhor analisar as interações ar-mar: um em $46^{\circ} \mathrm{S} 057^{\circ} \mathrm{W}$ (no Sudoeste do domínio) e outro em 31S048W.

No primeiro ponto $\left(46^{\circ} \mathrm{S} 057^{\circ} \mathrm{W}\right)$ além de estar próximo das medidas do navio, passaram dois ciclones consecutivos nas

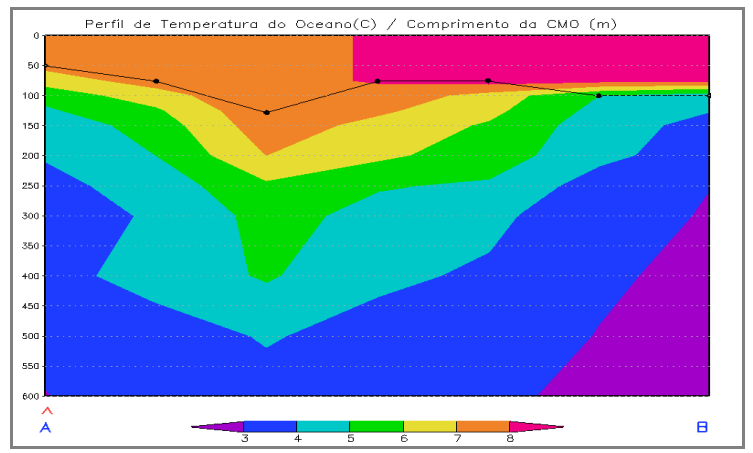

01NOV2004-06Z

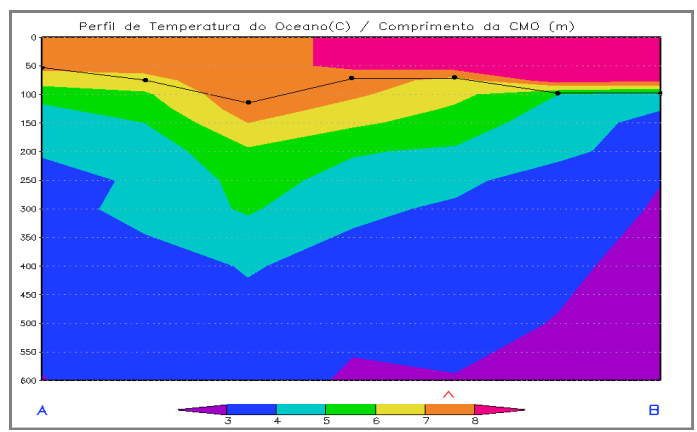

02NOV2004-06Z suas proximidades durante este período. Verificou-se que não houve precipitação (Figura 11), enquanto que no fluxo de calor sensível, as maiores diferenças ocorreram nos instantes de sinal positivo (fluxo para cima) e no calor latente caracterizou-se por ser o campo de maiores discrepâncias entre as simulações, chegando a valores duas vezes maiores que no caso controle. Estes fluxos estão de acordo com a estimativa de valores superiores da TSM originada pela CMO ativada no BRAMS com relação à climatológica.

$\mathrm{O}$ segundo ponto $\left(31^{\circ} \mathrm{S} 048^{\circ} \mathrm{W}\right)$ estava inicialmente sob o domínio de um centro de alta pressão e posteriormente (04NOV) uma frente estacionária, trazendo abaixamento de pressão e grande instabilidade para a região. Notou-se através do meteograma (Figura 12a) a ocorrência da precipitação convectiva nos dias 04 e 05 de maneira mais persistente no caso CMO ativa, entretanto, o caso controle apresentou maiores taxas. A TSM da simulação com a CMO ativa apresentou-se cerca de $2,0^{\circ} \mathrm{C}-2,5^{\circ} \mathrm{C}$ mais quente que o caso controle. Este fator foi relevante para que os termos referentes aos fluxos de calor sensível $(\mathrm{DH}=30 \mathrm{Wm}-2) \mathrm{e}$ latente (DLE $=100 \mathrm{Wm}-2)$, também tenham se comportado de forma mais intensa que o controle. Vale ressaltar que o calor latente não apresentou períodos negativos, enquanto que o sensível caracterizou-se menor que zero durante o dia $03 \mathrm{em}$ ambas as simulações. No gráfico de TSM pode ser observado um salto do valor inicial por volta de $16^{\circ} \mathrm{C}$

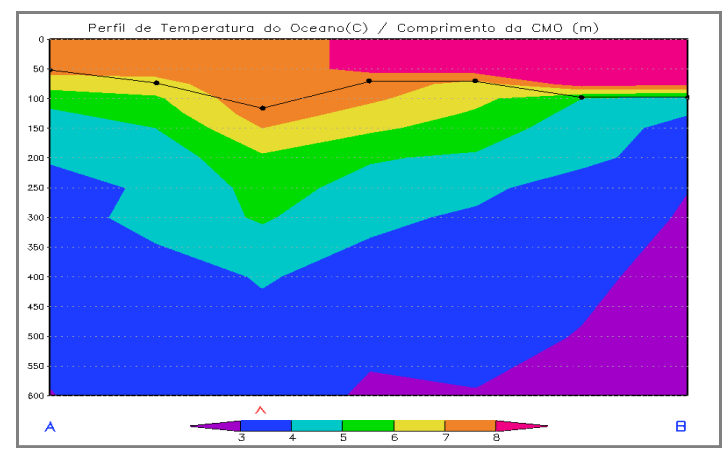

01NOV2004-18Z

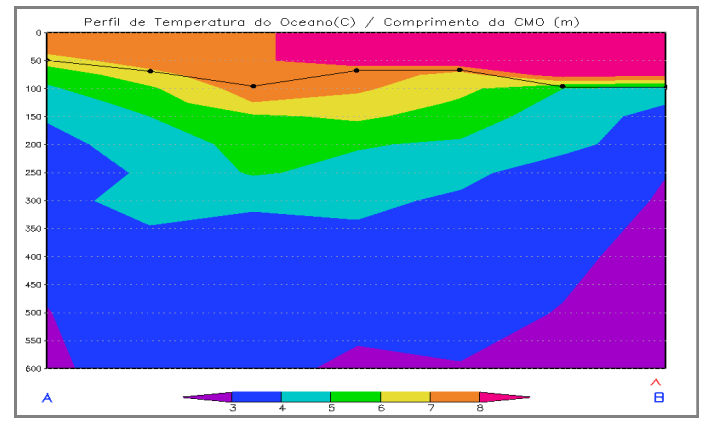

02NOV2004-18Z

Figura 8: Variação temporal do perfil da temperatura (em cores) na seção vertical da trajetória (AB) do primeiro ciclone. A seta em vermelho indica a posição do ciclone no instante considerado e a linha em preto indica a profundidade da CMO. 


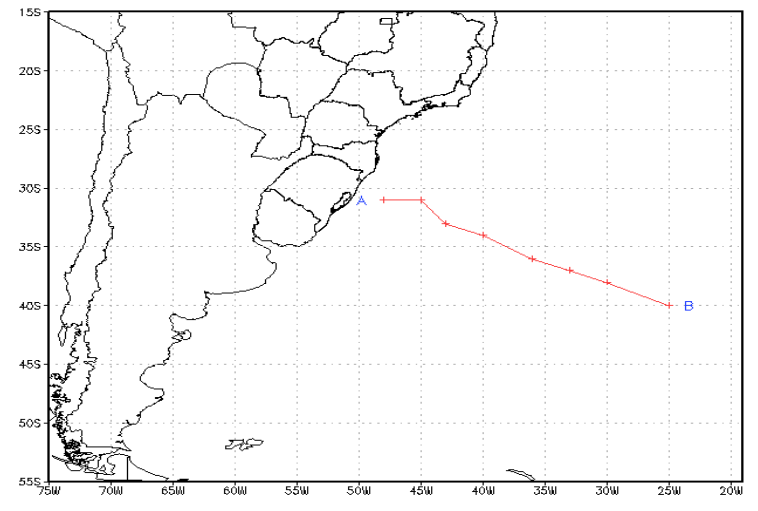

Figura 9: Trajetória do segundo ciclone com trajetória para SE.

para $18^{\circ} \mathrm{C}$ nos primeiros instantes, atribuído ao ajuste feito pelo modelo a uma resposta à submissão do perfil climatológico de temperatura do oceano a uma condição atmosférica realística.

A série temporal para a simulação com a $\mathrm{CMO}$ ativada no BRAMS (Figura 12b) apresentou inicialmente uma profundidade de $240 \mathrm{~m}$ e um diminuição gradativa até $180 \mathrm{~m}$, mantendo uma temperatura média de $18^{\circ} \mathrm{C}$. O HYCOM, entretanto, apresentou ciclos de maior variação com profundidades variando entre 130 $\mathrm{m}$ e $30 \mathrm{~m}$ nos dias 01 e 04 . Cabe mencionar que as diferenças entre as TSM não passam de $2,5^{\circ} \mathrm{C}$ e aumentando suavemente no decorrer do tempo.

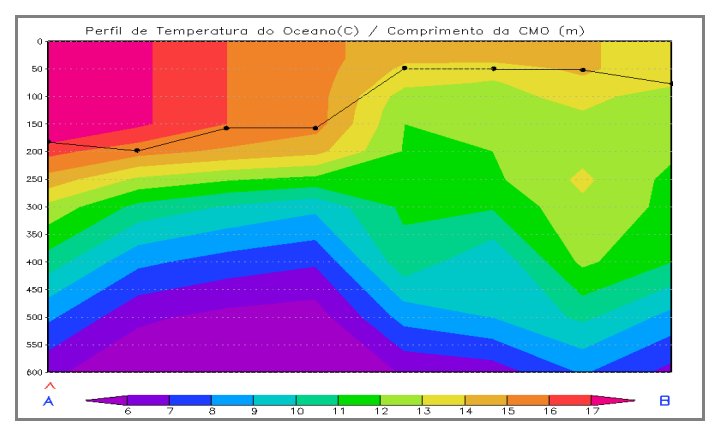

05NOV2004-18Z

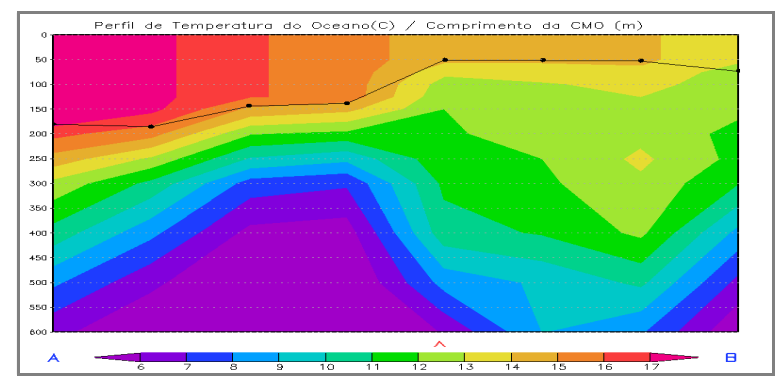

06NOV2004-18Z

\section{CONCLUSÕES}

O problema físico do trabalho foi comparar a evolução da TSM fornecida por uma máscara constante com outra resultante da variação da espessura da CMO.

Em primeiro lugar, modelo HYCOM obteve boa correlação com os dados coletados de XBT (temperatura e profundidade da $\mathrm{CMO}$ ), servindo de um bom indicativo para as comparações.

A TSM apresentou grande variação nos instantes iniciais, mantendo diferenças da ordem de $1,0^{\circ} \mathrm{C}-5,0^{\circ} \mathrm{C}$ em todo o domínio do modelo, independente do fenômeno atmosférico estudado, devido ao fato do ajuste das características do perfil da CMO da base climatológica LEVITUS ter divergido da TSM climatológica do BRAMS na maioria dos pontos, com evidência na região da CBM. Entretanto, uma vez ajustada a TSM, houve pouca variação no decorrer do tempo (em torno de $1,0^{\circ} \mathrm{C}-2,5^{\circ} \mathrm{C}$ ). Foi interessante também observar que apesar destas diferenças de TSMs, os fluxos de calor manifestaramnas de maneira efetiva e direta: quanto maior (menor) a TSM, maiores (menores) os fluxos de calores latente e sensível.

Outro fator relevante foi a relação da TSM com a profundidade da $\mathrm{CMO}$, conforme ocorreu o aprofundamento (estreitamento) da camada, a temperatura resfriou (aqueceu), mesmo que discretamente, de acordo com a relação dos ventos fracos de Chu (1993).

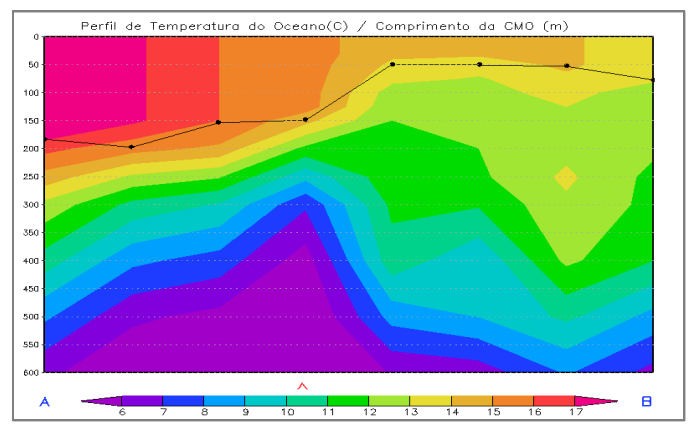

06NOV2004-12Z

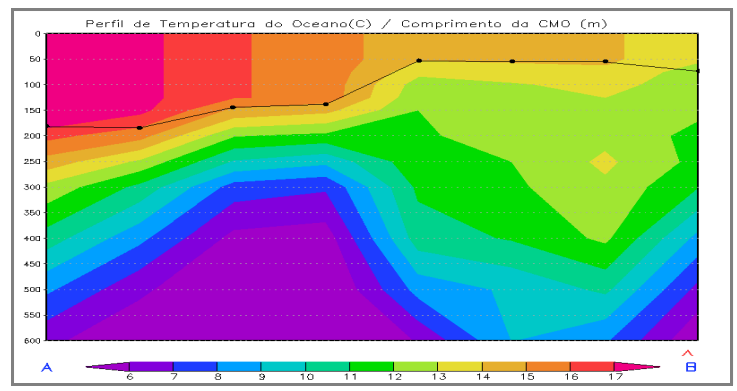

07NOV2004-12Z

Figura 10: Variação temporal do perfil da temperatura (em cores) na seção vertical da trajetória (AB) do segundo ciclone. A seta em vermelho indica a posição do ciclone no instante considerado e a linha em preto indica a profundidade da CMO. 


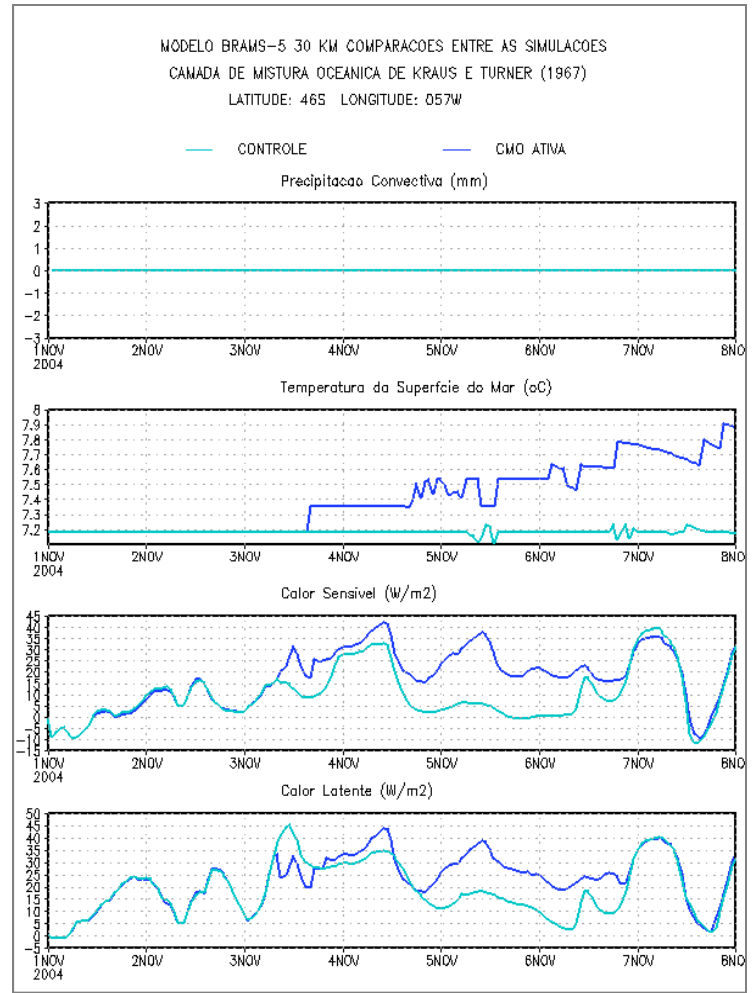

(a)

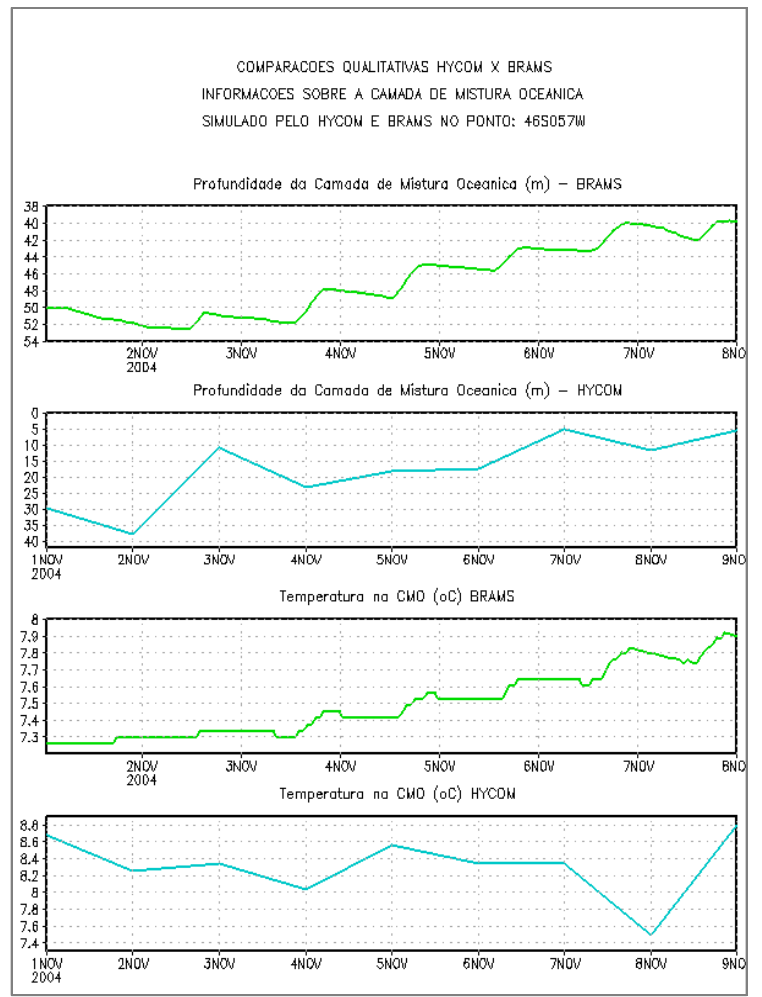

(b)

Figura 11: (a) Série temporal das comparações entre as variáveis atmosféricas dos modelos com TSM climatológica (controle) e CMO ativa; (b) variáveis oceânicas do Modelo CMO e do Modelo HYCOM no ponto $46^{\circ} \mathrm{S} 057^{\circ} \mathrm{W}$.

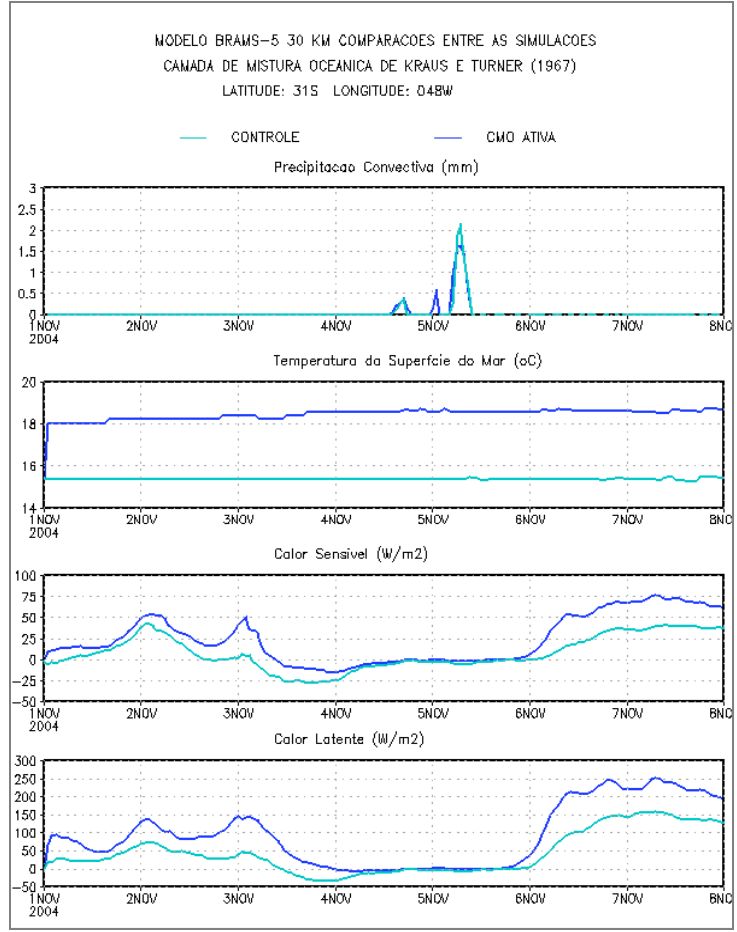

(a)

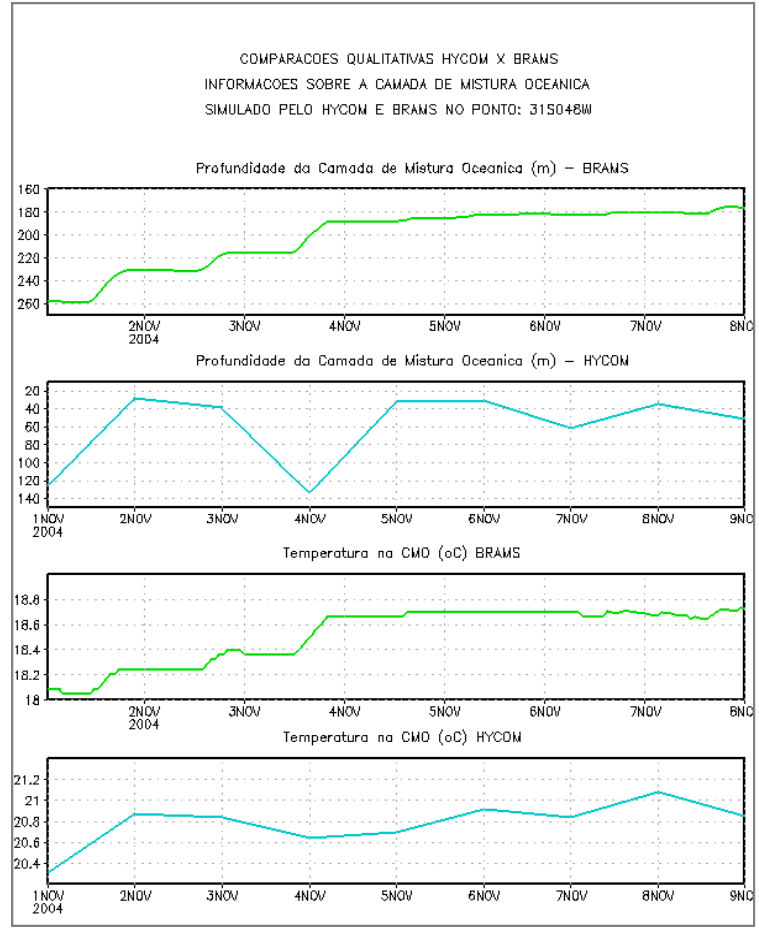

(b)

Figura 12: (a) Série temporal das comparações entre as variáveis atmosféricas dos modelos com TSM climatológica (controle) e CMO ativa; (b) variáveis oceânicas do Modelo CMO e do Modelo HYCOM no ponto $31^{\circ} \mathrm{S} 048^{\circ} \mathrm{W}$. 
A CMO modelada pelo BRAMS caracterizou-se por estreitar durante as trajetórias das baixas pressões, embora não tenha indicado um significativo aprofundamento seguindo o anticiclone da retaguarda. Vale ressaltar que este fato de em média, ter ocorrido um estreitamento da CMO ativa, provavelmente devido a ausência do termo de produção de energia cinética turbulenta por cisalhamento da corrente.

As diferenças pontuais entre a CMO-BRAMS e a CMO-HYCOM mostraram que a espessura da CMO superestimada no perfil LEVITUS desviou das reanálises, comprometendo a análise direta entre as duas CMO, caracterizando-se um problema de valor inicial. Esta característica pareceu um fator limitante no uso desta metodologia, merecendo atenção.

Finalmente, vale sempre ressaltar que o modelo de $\mathrm{CMO}$ de K-T introduzido no BRAMS é termodinâmico e reagiu bem ao seu propósito. Os grandes aprofundamentos e resfriamentos, observados nas simulações do HYCOM, devem-se a fatores da circulação oceânica (advecção de por correntes, mistura pelo vento aumentando a ECT, bombeamento de Ekman, etc.). Para tamanha precisão seria necessário direcionar a pesquisa ao acoplamento de modelos atmosféricos e oceânicos. Fato completamente descartado neste trabalho.

\section{AGRADECIMENTOS}

Ao Dr. Ronald Buss de Souza, pesquisador da Divisão de Sensoriamento Remoto do INPE, pelos dados meteooceanográficos coletados durante o INTERCONF do Programa Antártico Brasileiro (CNPq/Proantar). Ao Dr. Edmilson Freitas pela contribuição na modelagem numérica com o BRAMS e à CAPES/CNPq pelo apoio financeiro a este trabalho.

\section{REFERÊNCIAS BIBLIOGRÁFICAS}

ADAMEC, D.; ELSBERRY R. L. Response of an intense oceanic current system to cross-stream cooling events. Journal of Physical Oceanography., v.15, p. 273-287, 1985a.

ADAMEC, D.; ELSBERRY R. L. The response of an intense oceanic current systems entering regions of strong cooling. Journal of Physical Oceanography, v.15, p.1284-1295, 1985 b.

BANE, J.M.; OSGOOD, K.E. Winter-time air-sea interaction processes across the Gulf Stream. Journal of Geophysical Research, v.94, p. 10755-10772, 1989.

BOSART, L. F.; LACKMANN, G. M. Post-landfall tropical cyclone reintensification in a weakly-baroclinic environment: A case study of hurricane David (September 1979). Monthly Weather Review, v.123, p. 3268-3291, 1995.
CANIAUX, G.; PLANTON, S. A 3D ocean mesoscale simulation using data from the SEMAPHORE experiment: mixed layer heat budget. Journal of Geophysical Research., v.103, n. C11, p. 25081-25099, 1998.

CAMPOS, E. J. D.; LENTINI, C. A. D.; MILLER, J. L.; PIOLA, A. R. Interannual variability of the sea surface temperature in the South Brazil Bight. Geophysical Research Letters, v.26, n. 14, p. 2061-2064, 1999.

CHU, P. C. Generation of low frequency unstable modes in a coupled equatorial troposphere and ocean mixed layer. Journal of Atmospheric Science, v. 50, p. 731-749, 1993.

DOBSON, F. W.; SMITHH, S. D. Bulk models of solar radiation at sea. Quarterly Journal of the Royal Meteorological Society., v. 114, p. 165-182, 1988.

DOYLE, J. D.; WARNER, T. T. Mesoscale coastal processes during GALE IOP2. Monthly Weather Review, v. 118, p. 283-308, 1990.

FAZENDA, A. L.; DEMERVAL, S. M.; ENARI, E. H.; PANETT, J.; RODRIGUES, L. F. First Time User's Guide (BRAMS Version 3.2). CPTEC/INPE. Cachoeira Paulista, 2006. 24p.

FRIEHE, C. A.; SHAW, W. J.; ROGERS, D. P.; DAVIDSON, K. L.; LARGE, W. G.; STAGE, S. A.; CRESCENTI, G. H.; KHALSA, S. J. S.; GREENHUT, G. K.; LI, F. Air-sea fluxes and surface layer temperatures around a sea-surface temperature front. Journal of Geophysical Research, v. 96, p. 8593-8609, 1991.

GIORDANI, H.; PLANTON, S.; BENECH, B.; KWON, B. H. Atmospheric Boundary Layer Response to Sea Surface Temperatures during the SEMAPHORE Experiment. Journal of Geophysical Research, v. 103, p. 25047-25060, 1998.

HUANG, R.. Does cooling drive the Gulf Stream recirculation? Journal of Physical Oceanography, v. 20, p. 750-757, 1990.

KHALSA, S. D. S. Atmospheric stability over the tropical oceans derived from TIROS Operational Vertical Sounder. Journal of Applied Meteorology and Climatology, v. 28, p. 1002-1009, 1989.

KRAUS, E. B.; TURNER, J. S. A one-dimensional model of the seasonal thermocline II, the general theory and its consequences. Tellus, v. 19, p. 98-105, 1967.

MCPHADEN, M. J. Variability in the central equatorial Indian Ocean. Part I: Ocean dynamics. Journal of Marine Research., v. 40, p. 157-176, 1982.

NOF, D. On the response of ocean currents to atmospheric cooling. Tellus, v. 35A, p. 60-72, 1983.

PEZZI, L. P.; SOUZA, R. B.; DOURADO, M. S.; MATA, M. M.; GARCIA, C. A. E.; DIAS, M. A. F. A. S. Oceanatmosphere in situ observations at the Brazil-Malvinas Confluence region. Geophysical Research Letters, v. 32, n. 22, p. 1-4, 2005. 
SARAIVA, J. M. B. Previsão de tempo na região sul: efeitos locais e influência da liberação de calor latente. 176p. 1996. Tese de Doutorado do Departamentode Ciências Atmosféricas do Instituto de Astronomia, Geofísica e Ciências Atmosféricas da Universidade de São Paulo. São Paulo. 1996.

SWEET, W.; FETT, R.; KERLING, J.; LAVIOLETTE, P. Airsea interaction effects in the lower troposphere across the north wall of the Gulf Stream. Monthly Weather Review, v. 109, p. 1042-1052, 1981.

WAINER, I.; VENEGAS, S. A. South Atlantic Multidecadal Variability in the Climate System Model. Journal of Climate, v. 15 , p. 1408-1420, 2002.
WAINER, I; TASCHETTO, A.; SOARES, J.; OLIVEIRA, A. P.; OTTO-BLIESNER, B.; BRADY, E. Intercomparison of Heat Fluxes in the South Atlantic. Part I: The Seasonal Cycle. Journal of Climate, v. 16, p. 706-714, 2003.

WEARE, B. C. Uncertainties in estimates of surface heat fluxes derived from marine reports over the tropical and subtropical oceans. Tellus, v. 41, p. 357-370, 1989.

WELLER, R. A.; BAUMGARTNER, M. F.; JOSEY, S. A.; FISCHER, A. S.; KINDLE, J. C. Atmospheric forcing in the Arabian Sea during 1994-1995: Observations and comparisons with climatology and models. Deep-Sea Research Part II: Tropical Studies in Oceanography, v. 45, p. 1961-1999, 1998. 